\title{
Effect of the Pruritus on the Quality of Life: A Preliminary Study
}

\author{
Irem Eren Erturk, M.D., Ozer Arican, M.D. ${ }^{1}$, Imran Kurt Omurlu, M.D. ${ }^{2}$, Necdet Sut, M.D. ${ }^{3}$ \\ Department of Dermatology and Venereology, Tekirdag State Hospital, Tekirdag, ${ }^{1}$ Department of Dermatology and Venereology, Trakya \\ University Medical Faculty, Edirne, ${ }^{2}$ Department of Biostatistics and Medical Informatics, Adnan Menderes University Medical Faculty, \\ Aydin, ${ }^{3}$ Department of Biostatistics and Medical Informatics, Trakya University Medical Faculty, Edirne, Turkey
}

Background: Pruritus affects the quality of life $(\mathrm{QoL})$ in the patients. However, little is known of the impacts of itching on the QoL in the literature. Objective: In this study, a questionnaire eliciting specific responses about pruritus was used to determine the effect of itching and its severity on QoL in chronic pruritic patients. Methods: One hundred ten adult patients with chronic pruritus were surveyed. Once itch severity had been self-reported by patients, together with Skindex-29, they have been given the 18-item Pruritusrelated Life Quality Index (PLQI) questionnaire, which was used to generated the new index. Results: Significant correlations have been found between the pruritus severity score of participants and their total Skindex-29 $(p<0.001)$ with its function, emotion and symptom subscale scores $(p$ $<0.001$ for each). Also, significant correlations were evident between total PLQI $(p<0.001)$ and its psychosocial negativities, difficulties in everyday activities and social live subscale scores ( $p<0.001$ for each). In particular, the emotion subscale of Skindex-29 $(p=0.02)$ and psychosocial negativity subscale of PLQI $(p=0.02)$ were significantly high in psychopathologic patients. A highly positive statistical correlation between Skindex-29 and PLQI scales was evident $(r=0.845, p<0.001)$. For reliability, Cronbach's coefficient a coefficients were 0.931 for the Skindex-29 scale

Received April 12, 2011, Revised May 4, 2011, Accepted for publication August 19, 2011

Corresponding author: Ozer Arican, M.D., Department of Dermatology and Venereology, Trakya University Medical Faculty, Edirne, Turkey (TR-22000). Tel: 90-284-235-76-41/1282, Fax: 90-284-235-27-30, Email: ozerari@gmail.com

This is an Open Access article distributed under the terms of the Creative Commons Attribution Non-Commercial License (http:// creativecommons.org/licenses/by-nc/3.0) which permits unrestricted non-commercial use, distribution, and reproduction in any medium, provided the original work is properly cited. and 0.918 for the PLQI scale. Conclusion: The results demonstrate that chronic pruritus appreciably reduces the QoL. PLQI could be used in the development of new questionnaires for determining the QoL of patients with chronic itch. (Ann Dermatol 24(4) 406 412, 2012)

\section{-Keywords-}

Pruritus, Quality of life, Questionnaires

\section{INTRODUCTION}

Pruritus is an undesirable condition that stimulates itching, and it may affect unfavorably the sleep and the quality of life $(\mathrm{QoL})^{1-3}$. Its actual incidence in the population is not clearly understood ${ }^{2-4}$. In a study conducted in our country, the incidence of itching was reported as $60 \%$ for the last week among the patients admitted to a dermatology outpatient clinic in July ${ }^{4}$. While the incidence of itching in atopic dermatitis among the dermatologic disorders has been reported as $81 \sim 87 \%$, the incidence in psoriasis has varied more widely, from $41 \sim 96 \%{ }^{3,5,6}$. Furthermore, an incidence of $29 \%$ has been reported for pruritus in subjects over 50 -years-of-age ${ }^{5}$. Pruritus is related with many systemic diseases, psychological problems or use of drugs; if the condition exceeds 6 weeks it is considered to be chronic pruritus ${ }^{2,3}$.

The problems or diseases associated with skin affect only one organ, but can influence the psychosocial status of the patient, his/her social interactions, his/her daily activities and his/her $\mathrm{QoL}^{7}$. Pruritus affects primarily the QoL in pruritic dermatosis $^{8-10}$. Nonetheless, only a few studies have investigated the impacts of itching on the $\mathrm{QoL}^{11-13}$. In addition, only a single study published during the time of the present study has addressed QoL through the use of 
pruritus-focused questions ${ }^{14}$.

The present study aimed to investigate the impact of pruritus and its intensity on the QoL in patients with chronic pruritus by using a test comprising pruritus-focused questions.

\section{MATERIALS AND METHODS}

This cross-sectional definitive pattern study was designed in the Dermatology Department of the Trakya University Medical Faculty, Edirne, Turkey to identify the QoL of chronic pruritus patients. The study was approved by the local ethical committee of the medical faculty.

The data gathering stage of the study was conducted for one year starting from May 2008. Patients with chronic pruritus having itching complaints for more than 3 months and who were over 18-years-of-age were included. Patients were not grouped with respect to their diseases. Pregnant women and those not receiving topical or systemic therapies due to the itching occurring within the last 3 months were excluded. One hundred and twenty four successive patients were finally admitted for study. Of these, 110 agreed to participate.

Hemogram, preprandial blood glucose, renal function tests, hepatic enzymes, thyroid hormones and serum immunoglobulin $\mathrm{E}$ levels were determined in all patients in terms of the presence of the accompanying diseases. Furthermore, psychiatry consultation was requested for all patients. Information such as name, surname, age, gender, marital status, educational level, job/profession, dermatological diagnosis, duration of disease, age at diagnosis, age at start of therapy, whether he/she received therapy regularly, therapies currently administered (if any), presence of an additional accompanying disease, and presence of any psychiatric disorder were recorded.

Subsequently, the study questionnaire was given to the participants together with a text indicating the objective of the study. The patients answered the questionnaire items under the supervision of the attending physician. For the measurement of the QoL, the Pruritus-related Life Quality Index (PLQI) that was newly-developed by the authors, and the Skindex- $29^{15}$ were used (Table 1). The PLQI had been pilot-tested on a small patient group before beginning the study. The PLQI questionnaire comprised 18 items and the response options (none, a little, fairly, and too much) were respectively scored as $0,1,2$, and 3 . Questions 1-6, 9, 11, and 13 15 evaluated psychosocial negativity, questions 16 18 evaluated therapy, and questions $7,8,10$, and 12 evaluated difficulties in daily life. In the Skindex-29 questionnaire, 29 questions were posed. The answer options were gathered into five classes (never, rarely, sometimes, frequently, and always) and were respectively scored as $0,25,50,75$, and 100 points. The questions were also evaluated using function, symptom, and emotion subscales. The participants were eventually requested to make a preference between the itching scores ${ }^{16}$. The scale used in evaluating the itching score also constituted of 4 options: (0)-none, (1)-slight (sometimes, slight itching), (2)-moderate (persistent or intermittent itching not disturbing sleep) and (3)-severe (irritative itching disturbing sleep).

\section{Analyses}

The data were reviewed and statistical analyses were performed using the Minitab (Minitab Inc., State College, PA, USA) statistical package program (Serial Number: WCP131.00197). The conformability of the quantitative data to the normal distribution was examined with Kolmogorov-Smirnov test. Since the age and the initial age variables were distributed normally, the descriptive statistics were presented as mean \pm standard deviation. In the comparison between itching, which is not in concordance with normal distribution, for total Skindex-29 and the subscales of this, and between PLQI scores and the

Table 1. The questions included into the Pruritus-Related Life Quality Index

1. I feel that other people are staring at me because of my itching.

2. Other people ask me about my itching.

3. I think that I may propagate my itching to other people.

4. People do not want to eat the meals that I cook due to my itching.

5. I could not go beside the people that I do not recognize due to my itching.

6. I am ashamed of being obliged of itching beside other people.

7. I could not wear any clothes that I desire due to my itching.

8. I abstain to go to the hairdresser due to my itching.

9. People care not to touch me (handshaking, etc.) due to my itching.

10. I have difficulties in finding job due to my itching.

11. Since they think that my itching is contaminating people keep away from me and they do not want to be in same medium with me.

12. Because of my itching, although I want go to, I abstain to go places (shopping, beach, restaurant, café, and invitations) where the people are gregarious.

13. Because of my itching, I have trouble getting close to the opposite sex and, (if single) I think that I will not be able to get married.

14. Because of my itching, my clothes get dirty more frequently.

15. My sexual life is affected negatively because of my itching.

16. Using topical drugs because of my itching seems to me annoying and time consuming.

17. I think that the drugs I use may have side effects.

18. I think the pruritus therapies very expensive for me.

Options for each question are as: 1. None, 2. A little, 3. Fairly and 4. Too much. 
subscales of this, the Mann-Whitney $U$ test was used in terms of number of groups. Furthermore, the descriptive statistics were shown as median (25\% to $75 \%)$. In the investigation of the relationships between the total Skindex-29 and its subscales, between total PLQI and its subscales, between the variables such as the itching score, and the initial age and time, Spearman Correlation analysis was used. Internal consistency reliability of the scales was assessed by calculating Cronbach's alpha coefficient. The statistical significant level was given with the concerning tests and once $p<0.05$ was obtained it was accepted as significant.

\section{RESULTS}

All 110 patients (66 females, 44 males) answered all the questions of the questionnaire. The mean \pm standard deviation age of the participants was $49 \pm 15$ years (18 86 years). Ninety one of the participants were married and the remaining 19 were single. Nine of the participants were illiterate, 69 were primary school graduates, 23 were high school graduates, and nine were university graduates.

Forty six of the subjects had another systemic disease, 27 displayed a psychiatric pathology and 33 were receiving medication due to these additional conditions. The cases that were included into the study comprised patients with the diagnoses of idiopatic pruritus $(n=45,40.91 \%)$, chronic urticaria $(n=16,14.55 \%)$, erythemato-squamous diseases $(n=17,15.45 \%)$, diffuse superficial mycoses and id reactions associated with these $(n=20,18.18 \%)$, and other pruritic skin diseases $(n=12,10.91 \%)$. The statistical data between diagnosis and QoL are shown in Table 2. Only the function subscale of Skindex-29 in erythemato-squamous skin diseases was different, being slightly more affected than the other subscales. There was no difference in the total and other subscales of both questionnaires for the various pruritic skin diseases. Eight of these patients had slight itching, 38 had moderate itching and 64 had severe itching. Most $(77.3 \%)$ patients had been affected by itching for longer than 12 months (2 months $\sim 39$ years, mean $=45 \pm 6$ months).

The female/male ratio was $3 / 2$. No statistical difference was observed $(p>0.05)$ between the 2 genders in terms of Skindex-29 with its subscales and PLQI with itching scores. No statistical association was found between the ages of the patients and total Skindex-29, total PLQI, and the subscales of these and the itching scores $(p>0.05)$. Concordantly, no statistical association was found in terms of the initial age of the disease, total Skindex-29, total PLQI, and itching scores $(p>0.05)$. There was no relationship between the disease duration and subscales of QoL and the itching scores $(p>0.05)$.

The majority of the subjects (99/110, 82.7\%) were married. To facilitate the statistical evaluation, we included the widowed, divorced and those living separately in

Table 2. The influence of etiology of pruritus on the quality of life and on the pruritus

\begin{tabular}{|c|c|c|c|c|c|c|c|}
\hline $\begin{array}{l}\text { Questio- } \\
\text { nnaire }\end{array}$ & Scale & $\begin{array}{l}\text { Idiopatic pruritus } \\
\qquad(\mathrm{n}=45)\end{array}$ & $\begin{array}{l}\text { Chronic urticaria } \\
\quad(n=16)\end{array}$ & $\begin{array}{l}\text { Erythemato- } \\
\text { squamous skin } \\
\text { diseases } \\
(n=17)\end{array}$ & $\begin{array}{l}\text { Superficial } \\
\text { mycosis } \\
(n=20)\end{array}$ & $\begin{array}{l}\text { Other dermatitis } \\
\quad(n=12)\end{array}$ & $p$-value \\
\hline \multirow[t]{4}{*}{ Skindex-29 } & Function & $\begin{array}{c}41.67 \\
(30.21 \sim 50.00)\end{array}$ & $\begin{array}{c}50.00 \\
(36.46 \sim 57.81)\end{array}$ & $\begin{array}{c}54.17 \\
(35.42 \sim 62.50)\end{array}$ & $\begin{array}{c}40.63 \\
(27.08 \sim 45.83)\end{array}$ & $\begin{array}{c}53.13 \\
(40.10 \sim 61.98)\end{array}$ & $0.03^{\dagger}$ \\
\hline & Symptom & $\begin{array}{c}42.86 \\
(35.71 \sim 53.57)\end{array}$ & $\begin{array}{c}50.00 \\
(33.93 \sim 57.14)\end{array}$ & $\begin{array}{c}46.43 \\
(32.14 \sim 58.93)\end{array}$ & $\begin{array}{c}48.21 \\
(30.35 \sim 53.57)\end{array}$ & $\begin{array}{c}50.00 \\
(30.36 \sim 57.14)\end{array}$ & 0.82 \\
\hline & Emotion & $\begin{array}{c}40.00 \\
(26.25-51.25)\end{array}$ & $\begin{array}{c}51.25 \\
(33.75 \sim 58.75)\end{array}$ & $\begin{array}{c}50.00 \\
(37.50 \sim 55.00)\end{array}$ & $\begin{array}{c}38.75 \\
(28.13 \sim 51.88)\end{array}$ & $\begin{array}{c}46.25 \\
(34.38 \sim 60.63)\end{array}$ & 0.29 \\
\hline & Total & $\begin{array}{c}42.24 \\
(29.74 \sim 49.14)\end{array}$ & $\begin{array}{c}50.43 \\
(39.00 \sim 56.25)\end{array}$ & $\begin{array}{c}51.72 \\
(38.36 \sim 57.32)\end{array}$ & $\begin{array}{c}42.67 \\
(27.59 \sim 49.57)\end{array}$ & $\begin{array}{c}50.43 \\
(34.91 \sim 62.93)\end{array}$ & 0.14 \\
\hline \multirow[t]{4}{*}{ PLQI } & $\begin{array}{c}\text { Psychosocial } \\
\text { negativities }\end{array}$ & $\begin{array}{c}13.00 \\
(8.50 \sim 20)\end{array}$ & $\begin{array}{c}17.50 \\
(13.25 \sim 22.00)\end{array}$ & $\begin{array}{c}18.00 \\
(9.50 \sim 21.00)\end{array}$ & $\begin{array}{c}16.50 \\
(8.25 \sim 19.75)\end{array}$ & $\begin{array}{c}16.50 \\
(14.00 \sim 20.75)\end{array}$ & 0.41 \\
\hline & Treatment & $\begin{array}{c}4.00 \\
(2.00 \sim 6.00)\end{array}$ & $\begin{array}{c}5.00 \\
(4.25 \sim 6.00)\end{array}$ & $\begin{array}{c}5.00 \\
(3.00 \sim 6.00)\end{array}$ & $\begin{array}{c}4.50 \\
(3.00 \sim 6.00)\end{array}$ & $\begin{array}{c}5.00 \\
(2.00 \sim 6.75)\end{array}$ & 0.46 \\
\hline & $\begin{array}{l}\text { Difficulties } \\
\text { in daily life }\end{array}$ & $\begin{array}{c}6.00 \\
(3.00 \sim 7.50)\end{array}$ & $\begin{array}{c}6.50 \\
(4.25 \sim 9.00)\end{array}$ & $\begin{array}{c}8.00 \\
(5.00 \sim 8.00)\end{array}$ & $\begin{array}{c}5.00 \\
(4.00 \sim 6.75)\end{array}$ & $\begin{array}{c}6.50 \\
(2.00 \sim 6.75)\end{array}$ & 0.12 \\
\hline & Total & $\begin{array}{c}24.00 \\
(13.50 \sim 35.00)\end{array}$ & $\begin{array}{c}33.50 \\
(25.50 \sim 37.00)\end{array}$ & $\begin{array}{c}32.00 \\
(19.50 \sim 37.00)\end{array}$ & $\begin{array}{c}27.00 \\
(17.00 \sim 34.50)\end{array}$ & $\begin{array}{c}6.50 \\
(3.50 \sim 8.00)\end{array}$ & 0.24 \\
\hline \multicolumn{2}{|c|}{ Itching score } & $3(2 \sim 3)$ & $3(2 \sim 3)$ & $3(2 \sim 3)$ & $3(2 \sim 3)$ & $3(2 \sim 3)$ & \\
\hline
\end{tabular}

Values are presented as median $\left(25 \sim 75 \%\right.$ percentile). PLQI: Pruritus-related Life Quality Index. Kruskal Wallis test, ${ }^{\dagger}$ statistically significant with $p$-values $<0.05$. 
Table 3. The influence of the psychiatric state on the quality of life and on the pruritus

\begin{tabular}{llccc}
\hline \multicolumn{1}{c}{ Scale } & Absent $(\mathrm{n}=83)$ & Available $(\mathrm{n}=27)$ & $p$-value \\
\hline Skindex-29 & Function & $43.75(33.33 \sim 52.08)$ & $50.00(37.50 \sim 60.41)$ & 0.09 \\
& Symptom & $42.85(32.14 \sim 53.57)$ & $50.00(35.71 \sim 64.28)$ & 0.07 \\
& Emotion & $42.50(30.00 \sim 52.50)$ & $50.00(30.00 \sim 60.00)$ & $0.02^{\dagger}$ \\
& Total & $43.96(32.75 \sim 50.86)$ & $51.72(34.48 \sim 62.06)$ & $0.02^{\dagger}$ \\
PLQI & Psychosocial negativities & $16.00(10.00 \sim 21.00)$ & $22.00(7.00 \sim 24.00)$ & $0.02^{\dagger}$ \\
& Treatment & $5.00(3.00 \sim 6.00)$ & $7.00(3.00 \sim 9.00)$ & 0.18 \\
& Difficulties in daily life & $6.00(3.00 \sim 8.00)$ & $7.00(3.00 \sim 8.00)$ & 0.21 \\
Itching & $27.00(17.00 \sim 33.00)$ & $36.00(13.00 \sim 38.00)$ & $0.02^{\dagger}$ \\
\hline
\end{tabular}

Values are presented as median (25 75\% percentile). PLQI: Pruritus-related Life Quality Index. Mann-Whitney $\cup$ Test, ${ }^{\dagger}$ statistically significant with $p$-values $<0.05$.

Table 4. The relationship between the itching score and quality of life

\begin{tabular}{clcc}
\hline \multirow{2}{*}{ Questionnaire } & \multicolumn{1}{c}{ Scale } & \multicolumn{2}{c}{ Itching score } \\
\cline { 3 - 4 } & & $r$ & $p$-value \\
\hline \multirow{2}{*}{ Skindex-29 } & Function & 0.62 & $<0.001^{\dagger}$ \\
& Symptom & 0.66 & $<0.001^{\dagger}$ \\
& Emotion & 0.59 & $<0.001^{\dagger}$ \\
& Total & 0.67 & $<0.001^{\dagger}$ \\
& Psychosocial & 0.71 & $<0.001^{\dagger}$ \\
& negativities & .0 .50 & $<0.001^{\dagger}$ \\
& Treatment & 0.50 & $<0.001^{\dagger}$ \\
& Difficulties & 0.59 & $<0.001^{\dagger}$ \\
\hline
\end{tabular}

PLQI: Pruritus-related Life Quality Index, r: Spearman correlation coefficient. ${ }^{\dagger}$ Statistically significant with $p$-values $<0.001$.

the category of singles and those living together into the married category. No difference was found between the 2 marital categories in terms of Skindex-29 and PLQI, and their subscales and the itching scores $(p>0.05)$.

A psychiatric diagnosis (e.g., depression, anxiety, adjustment disorder) was made in 27 people (24.5\%) and no psychopathology was observed in the other 83 people $(75.5 \%)$. The statistical evidence between psychiatric state and QoL is summarized in Table 3. The emotion subscale of the patients $(p=0.02)$ with total Skindex-29 scores $(p=0.02)$ was significant in those with a psychiatric diagnosis. Also, when evaluated in terms of PLQI, statistically significant differences were found in the total PLQI scores $(p=0.02)$ and in the subscale of psychosocial negativity of these $(p=0.02)$. No significant difference $(p=0.23)$ was found between the 2 groups in terms of itching scores.

When the state of systemic disease was studied in the participants of study, it was seen that there was not any
Table 5. Cronbach- $\alpha$ coefficients of Skindex-29 and PLQI scales

\begin{tabular}{lc}
\hline Questionnaires and scales & Cronbach $\alpha$ coefficient* \\
\hline Total Skindex-29 & 0.931 \\
Function & 0.868 \\
Symptom & 0.743 \\
Emotion & 0.812 \\
Total PLQI & 0.918 \\
Psychosocial negativities & 0.893 \\
Treatment & 0.592 \\
Difficulties in daily life & 0.701 \\
\hline
\end{tabular}

*Reliability analysis, PLQI: Pruritus-related Life Quality Index.

additional disease in 64 people $(58.1 \%)$, with at least one systemic disease including diabetes mellitus, hypertension, hyperthyroidism, hypothyroidism, hyperlipidemia, hypercholesterolemia, and chronic renal failure evident in 46 patients $(41.9 \%)$. No statistically significant difference $(p>0.05)$ was found between both the QoL scale and itching scores according to the presence of any systemic disease.

Significant correlations in advanced level were found between the itching score and Skindex-29 and its subscales, PLQI and its subscales with the itching scores. The relationship between the itching score of the participants and the QoL scores is provided in Table 4.

\section{Reliability}

Cronbach's coefficient $\alpha$ coefficients were 0.931 for the Skindex-29 scale and 0.918 for the PLQI scale (Table 5), indicating good internal consistency reliability of the scales.

\section{Validity}

The graphical representations of the rank correlation of Skindex-29 score with PLQI score is shown in Fig. 1. 


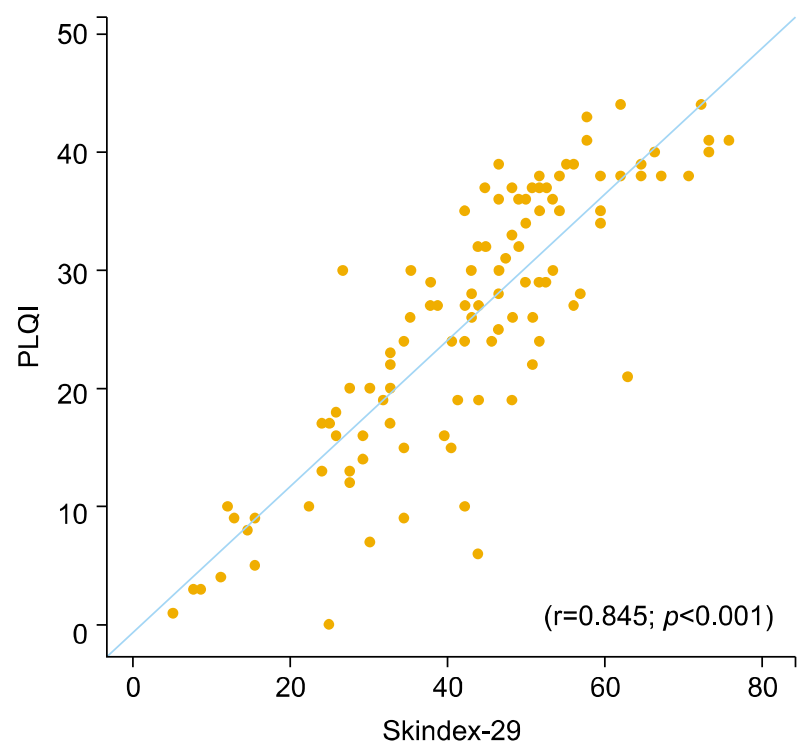

Fig. 1. Spearman rank correlation coefficients of Skindex-29 score with Pruritus-related Life Quality Index (PLQI) score.

Skindex-29 score significantly correlated with PLQI score $(r=0.845, p<0.001)$. The PLQI score increased while the Skindex-29 score increased.

\section{DISCUSSION}

Pruritus is an uncomfortable subjective sensation that can be expressed as burning sensation, numbness, and pain. The malady is an established dermatological and systemic disease, and can be relieved by scratching ${ }^{17}$. Although it is difficult to define, it is obvious that it can be annoying when it is chronic and that it affects the patients more and exponentially when the therapy fails. It has been reported that in dermatological diseases the severity of the pruritus is also more with psychosocial effect of the disease ${ }^{18,19}$.

Females have more complaints of pruritus compared to males $^{5,20,21}$. This was also found presently. This may reflect that complaints of pruritus are influenced by female-related hormonal and psychological or that female patients are more apt to disclose their condition to a physician. The frequency of pruritus or its severity may increase with age ${ }^{20,22}$. Dryness of the skin plays an important role. Presently, marital status, age at diagnosis, duration of disease, and the presence of accompanying systemic disease did not affect Skindex-29, PLQI, and the itching scores. Significantly higher total Skindex-29 scores were evident in those with psychiatric diagnosis, with the emotion subscale being especially affected. When evaluated in terms of PLQI, statistically significantly higher scores were evident in those with psychiatric problems compared to those without psychiatric problems. From this standpoint, psychosocial negativities were more influential among the test subgroups. In 2 distinct studies performed on the patients with pruritus, it was similarly demonstrated that pruritus increases the psychosocial morbidity of the patients ${ }^{5,23}$. Evidence of depression in patients with chronic pruritus compared to healthy control has been reported ${ }^{24}$. In other evaluations performed on the patients with psychogenic pruritus, the presence of depression and/or anxiety in addition to the personality disorder in these patients was noted ${ }^{25}$. In our study, it was not investigated whether the disease led to the psychiatric problem or whether pruritus developed following an underlying primary psychopathology. However, regardless of the etiology, in the patients whose psychological state is disturbed more or in those with already disturbed psychological state, QoL is affected negatively in more advanced level, independent of the pruritus severity. This can be also clearly seen from the fact that both tests were affected more in the emotion and psychosocial subscales, independent of the pruritus score. Therefore, regardless of the cause and the severity of the disease, more successful therapy can be provided by performing the psychiatric examination and by addressing the treatment with multidisciplinary approach and that more rapid improvement in the QoL of the patients.

In dermatoses such as atopic dermatitis and psoriasis, the fact that the pruritus progresses more severely in relation with the severity of the disease or is associated with the stress has been documented ${ }^{26-30}$. Weisshaar et al. ${ }^{30}$ demonstrated that chronic pruritus is an important and major factor influencing the QoL of dermatological patients. In a study on patients with chronic urticaria, the importance of pruritus on QoL was emphasized ${ }^{31}$. Whatever the etiology, this study showed that the effect on the QoL of pruritic diseases was not different from each other. Thus, it can be suggested that this symptom may worsen the QoL of the patient when prominent primarily or secondarily.

Despite the presence of many quality scales in dermatology, no questionnaire has been designed that indicates the effect of the pruritus and treatment on patient QoL. So, we preferred to use Skindex- $29^{15}$ as the standard test and a new test, PLQI, in Turkish. We found that as the itching score increases, not only its subscales increased but also the scores of Skindex-29 and PLQI increased. Furthermore, Skindex-29 and PLQI scores were correlated to each other. Desai et al. ${ }^{14}$ pilot examined a test (ItchyQoL) focusing on pruritus in 2008. In this test, the influence of the pruritus on the life of the patient was revealed by 27 questions with 5 response options, of which 22 were specific to pruritus. They performed this test similar to the other tests by considering responses under the subtitles of 
symptoms (6 questions), function (11 questions) and emotion (10 questions), and made assessments in terms of the gender and the diagnosis. The results supported the view that this test is more sensitive than Skindex-29 and Skindex-16. These authors also reported that the severity of pruritus could be gauged, especially by the function and emotion subsections, and that the frequency of pruritus is influenced with each of the three subsections, and concluded that their results should be supported by the studies that will be done with larger series.

Regardless of cause, there is an inverse proportion between the severity of pruritus and the QoL of the patient. In this context, the assessment of the psychosocial damage caused by the pruritus being an important symptom in the dermatology on the patient will enable a more objective evaluation through the development of the pruritus-focused QoL scales. We would to open a new way with our small initial study on this topic that we have observed as insufficient in the literature. In an uncontrolled study in limited number of pruritic patients, while only the patients admitting to the outpatient of dermatology department were considered, no distinction was made in the etiology and no comparison was made between the status before the treatment and after the treatment. We expect that the relationship between the pruritus and the QoL will be continued to be studied through the more extensive new controlled and comparative studies with multidisciplinary design, with larger populations by separating the etiologic causes, by using questionnaires such as PLQI or ItchyQoL.

\section{REFERENCES}

1. Grundmann S, Ständer S. Chronic pruritus: clinics and treatment. Ann Dermatol 2011;23:1-11.

2. Arican O. Mechanisms and treatment options of pruritus associated with systemic diseases: a review. Turkiye Klinikleri J Med Sci 2004;24:664-671.

3. Arican O. Pathophysiology, clinical presentation and management of pruritus. Turkderm 2005;39:88-97.

4. Dogramaci AC, Serarslan G. The prevalence of pruritus in Mustafa Kemal University Dermatology outpatient clinic during July 2008. Trakya Univ Tip Fak Derg 2009;26:137140.

5. van Os-Medendorp H, Eland-de Kok PC, Grypdonck M, Bruijnzeel-Koomen CA, Ros WJ. Prevalence and predictors of psychosocial morbidity in patients with chronic pruritic skin diseases. J Eur Acad Dermatol Venereol 2006;20:810-817.

6. Bilac C, Ermertcan AT, Bilac DB, Deveci A, Horasan GD. The relationship between symptoms and patient characteristics among psoriasis patients. Indian J Dermatol Venereol Leprol 2009;75:551.

7. Gurel MS, Yanik M, Simsek Z, Kati M, Karaman A. Quality of life instrument for Turkish people with skin diseases. Int J
Dermatol 2005;44:933-938.

8. Lange S, Zschocke I, Seidenglanz K, Schiffler A, Zollinger A, Amon $U$, et al. Predictors of the quality of life in patients with atopic dermatitis. Dermatol Psychosom 2000;1:66-70.

9. Gupta MA, Gupta AK, Schork NJ, Ellis CN. Depression modulates pruritus perception: a study of pruritus in psoriasis, atopic dermatitis, and chronic idiopathic urticaria. Psychosom Med 1994;56:36-40.

10. Duque MI, Yosipovitch G, Chan YH, Smith R, Levy P. Itch, pain, and burning sensation are common symptoms in mild to moderate chronic venous insufficiency with an impact on quality of life. J Am Acad Dermatol 2005;53:504-508.

11. Arican O. Measuring of quality of life in dermatological patients. Turkiye Klinikleri J Dermatol (Special Topics) 2009; 2:107-114.

12. Tessari G, Dalle Vedove C, Loschiavo C, Tessitore N, Rugiu C, Lupo A, et al. The impact of pruritus on the quality of life of patients undergoing dialysis: a single centre cohort study. J Nephrol 2009;22:241-248.

13. Tol H, Atalay $H$, Guney I, Gokbel $H$, Altintepe $L$, Buyukbas $S$, et al. The effect of Gabapentin therapy on pruritus, quality of life, depression and sleep quality in pruritic hemodialysis patients. Trakya Univ Tip Fak Derg 2010;27:1-5.

14. Desai NS, Poindexter GB, Monthrope YM, Bendeck SE, Swerlick RA, Chen SC. A pilot quality-of-life instrument for pruritus. J Am Acad Dermatol 2008;59:234-244.

15. Aksu AE, Urer MS, Sabuncu I, Saracoglu ZN, Chren MM. Turkish version of Skindex-29. Int J Dermatol 2007;46:350355.

16. Kaufmann R, Bieber $T$, Helgesen $A L$, Andersen $B L$, Luger $T$, Poulin $\mathrm{Y}$, et al; Multicentre investigator group. Onset of pruritus relief with pimecrolimus cream $1 \%$ in adult patients with atopic dermatitis: a randomized trial. Allergy 2006;61: 375-381.

17. Gieler U, Niemeier V, Brosig B, Kupfer J. Psychosomatic aspects of pruritus. Dermatol Psychosom 2002;3:6-13.

18. Amatya B, Nordlind K. Focus groups in Swedish psoriatic patients with pruritus. J Dermatol 2008;35:1-5.

19. Zachariae R, Zachariae CO, Lei U, Pedersen AF. Affective and sensory dimensions of pruritus severity: associations with psychological symptoms and quality of life in psoriasis patients. Acta Derm Venereol 2008;88:121-127.

20. Weisshaar E, Dalgard F. Epidemiology of itch: adding to the burden of skin morbidity. Acta Derm Venereol 2009;89:339350.

21. Reich A, Szepietowski JC, Wisnicka B, Pacan P. Does stress influence itching in psoriatic patients? Dermatol Psychosom 2003;4:151-155.

22. Yalçin B, Tamer E, Toy GG, Oztaş P, Hayran M, Alli N. The prevalence of skin diseases in the elderly: analysis of 4099 geriatric patients. Int J Dermatol 2006;45:672-676.

23. Schneider G, Driesch G, Heuft G, Evers S, Luger TA, Ständer S. Psychosomatic cofactors and psychiatric comorbidity in patients with chronic itch. Clin Exp Dermatol 2006;31:762-767.

24. Sheehan-Dare RA, Henderson MJ, Cotterill JA. Anxiety and depression in patients with chronic urticaria and generalized pruritus. Br J Dermatol 1990;123:769-774. 
25. Radmanesh M, Shafiei S. Underlying psychopathologies of psychogenic pruritic disorders. Dermatol Psychosom 2001; 2:130-133.

26. Chrostowska-Plak D, Salomon J, Reich A, Szepietowski JC. Clinical aspects of itch in adult atopic dermatitis patients. Acta Derm Venereol 2009;89:379-383.

27. Reich A, Szepietowski JC, Wisnicka B, Pacan P. Does stress influence itching in psoriatic patients? Dermatol Psychosom 2003;4:151-155.

28. Holm EA, Wulf HC, Stegmann H, Jemec GB. Life quality assessment among patients with atopic eczema. Br J Dermatol 2006;154:719-725.
29. Wittkowski A, Richards HL, Griffiths CE, Main CJ. The impact of psychological and clinical factors on quality of life in individuals with atopic dermatitis. J Psychosom Res 2004; 57:195-200

30. Weisshaar E, Apfelbacher C, Jäger G, Zimmermann E, Bruckner $\mathrm{T}$, Diepgen TL, et al. Pruritus as a leading symptom: clinical characteristics and quality of life in German and Ugandan patients. Br J Dermatol 2006;155:957-964.

31. Baiardini I, Pasquali M, Braido F, Fumagalli F, Guerra L, Compalati $\mathrm{E}$, et al. A new tool to evaluate the impact of chronic urticaria on quality of life: chronic urticaria quality of life questionnaire (CU-QoL). Allergy 2005;60:1073-1078. 